\title{
A Network Science Approach for Determining the Ancestral Phylum of Bacteria
}

\author{
Ehdieh Khaledian \\ School of Electrical Engineering and Computer Science, \\ Washington State University \\ Pullman, Washington \\ ehdieh.khaledian@wsu.edu \\ Kelly A. Brayton \\ Paul G. Allen School for Global Animal Health, \\ Department of Veterinary Microbiology Pathology, \\ Washington State University \\ Pullman, Washington, USA \\ kbrayton@wsu.edu
}

\author{
Assefaw H. Gebremedhin \\ School of Electrical Engineering and Computer Science, \\ Washington State University \\ Pullman, Washington, USA \\ assefaw.gebremedhin@wsu.edu \\ Shira L. Broschat \\ School of Electrical Engineering and Computer Science, \\ Paul G. Allen School for Global Animal Health, \\ Department of Veterinary Microbiology Pathology, \\ Washington State University \\ Pullman, Washington, USA \\ shira@wsu.edu
}

\begin{abstract}
Perhaps the most important organizing principle in biology for bacteria is the tree of phyla. It represents the evolution of bacteria now living in virtually every environment. The availability of whole genome sequences has provided the opportunity to reconstruct a comprehensive view of the tree and to trace the shared ancestry among all bacteria that have been sequenced. However, most existing research has presented the tree of phyla without considering the ancestral phylum. The objective of this study is to find the ancestral phylum using a network science approach and exploiting the availability of a rich dataset of genomes. For the analysis, a network representing 210 organisms is created by clustering more than 700,000 protein sequences for 28 recognized phyla. A network of phyla is then extracted from the results which is examined using a breadth-first search algorithm and centrality measures to create a rooted tree from which the likely ancestral phylum is identified.
\end{abstract}

\section{CCS CONCEPTS}

- Computational Biology $\rightarrow$ Clustering proteins; • Tree of life $\rightarrow$ Tree of bacterial phyla; $\bullet$ Network Science $\rightarrow$ Centrality Measures;

\section{KEYWORDS}

Clustering protein sequences, network of bacterial phyla, network of organisms, tree of bacterial phyla, ancestral phylum

\section{ACM Reference Format:}

Ehdieh Khaledian, Assefaw H. Gebremedhin, Kelly A. Brayton, and Shira L. Broschat. 2018. A Network Science Approach for Determining the Ancestral

Permission to make digital or hard copies of all or part of this work for personal or classroom use is granted without fee provided that copies are not made or distributed for profit or commercial advantage and that copies bear this notice and the full citation on the first page. Copyrights for components of this work owned by others than ACM must be honored. Abstracting with credit is permitted. To copy otherwise, or republish, to post on servers or to redistribute to lists, requires prior specific permission and/or a fee. Request permissions from permissions@acm.org.

ACM-BCB'18, August 29-September 1, 2018, Washington, DC, USA

(C) 2018 Association for Computing Machinery.

ACM ISBN 978-1-4503-5794-4/18/08 . \$ \$15.00

https://doi.org/10.1145/3233547.3233575
Phylum of Bacteria. In ACM-BCB'18: 9th ACM International Conference on Bioinformatics, Computational Biology and Health Informatics, August 29September 1, 2018, Washington, DC, USA. ACM, New York, NY, USA, 6 pages. https://doi.org/10.1145/3233547.3233575

\section{INTRODUCTION}

Through the process of evolution, natural selection in different environments has resulted in the current diversity of organisms. Biologists are interested in reconstructing this evolutionary path. Phylogenetic trees provide a way to chronicle past evolutionary events and, thus, have become central to our understanding of the process of evolution [10]. By measuring the magnitude of differences between genomes, we can create a phylogenetic tree and understand the evolutionary distance between organisms, i.e., how long ago they shared a common ancestor. The prevailing theory is that all life on Earth evolved from a single common ancestor several billions of years ago [19].

Advancement in high-throughput genome sequencing techniques has led to much progress in phylogenetics over the last two decades. As greater numbers of complete genomes become available, up-todate phylogenetic analyses can be performed. Woese et al. were the first to propose a phylogenetic tree based on the $16 \mathrm{~S}$ ribosomal RNA sequence. This work led to the existence of a hidden third branch in the tree of life which is now known as the archaea [20]. Researchers have since published many phylogenetic analyses in each of the three branches of the tree of life using similar methods $[4,6,11]$. Delsuc et al. tried to reconstruct the evolutionary history of organisms based on their genomes. In addition to showing advances in the construction of phylogenetic trees, they indicated future challenges of the phylogenomic approach for resolving the main branches of the tree [6]. At the time of its publication the work of Ciccarelli et al. was one of the latest attempts to reconstruct the tree of life for all fully sequenced genomes [4]. Jarvis et al. performed a genome-scale phylogenetic analysis of 48 species representing all orders of Neoaves (the clade of all modern birds) using phylogenomic methods created to handle genome-scale data [11]. The latest version of the tree of life was expanded by Hug et al. in 2016. They aligned and concatenated a set of ribosomal protein sequences from 
each organism to construct the tree of life [10]. Schulz et al. used small subunit rRNA gene sequences from metagenomes and all available reference genomes to depict a balanced view of bacterial phylogenetic diversity [17].

The studies described above offered a phylogenetic tree that is either unrooted or else rooted by a hypothetical most recent common ancestor of all the organisms or by an outgroup. Furthermore, they did not use whole genome sequences to create their tree, whereas looking at the whole genome to identify the relationships between organisms can provide new insight. The objective of our study was to extract the ancestral phylum of bacteria using different network theoretic approaches to reconstruct the evolutionary history of organisms based on an analysis of their complete genomes. To accomplish this, more than 700,000 protein sequences deduced from 210 complete genomes were downloaded from the National Center for Biotechnology Information (NCBI). These protein sequences were clustered and a network of organisms was created from the clusters. Networks of phyla were then obtained from the organism network. The relationships of 28 recognized phyla for bacteria were studied using network science. Furthermore, the possible ancestral phylum was extracted from the network of phyla.

\section{METHODOLOGY}

In the following sections we describe in detail the methods that we employed to construct the tree of bacterial phyla.

\subsection{Data Collection and Clustering}

A dataset of 733,227 protein sequences deduced from 210 complete genome sequences was downloaded from NCBI. Molecular ecological techniques based on 16S rRNA gene sequences indicate that less than $30 \%$ of presumptive bacterial phyla have been cultured. According to the LPSN (List of Prokaryotic names with Standing in Nomenclature) [16] only 29 of 100 bacterial phyla contain cultivated representatives. Therefore, limited information is available for more than 70 candidate bacterial phyla. Hence, in this work the downloaded dataset only represented the NCBI database in proportion to the availability of the 29 major recognized phyla [16].

Furthermore, four bacterial phyla account for most of the cultivated bacteria: Actinobacteria, Bacteroidetes, Firmicutes, and Proteobacteria while the remaining 25 bacterial phyla represent only a small portion. In fact, there is no complete genome available at NCBI for the Lentisphaerae phylum. As Proteobacteria are overrepresented in the database (> 50\%) and they are not monophyletic [2], classes of this phylum including Gammaproteobacteria, Alphaproteobacteria, Betaproteobacteria, Delta/Epsilon subdivision, and Zetaproteobacteria were considered separately. As a result, a total of 31 nodes were represented in our network rather than 28 , which included five classes of Proteobacteria, the Bacteroidetes/Chlorobi group (the two phyla are combined by NCBI) as one node, and the remaining 25 phyla. Because we have split phyla and merged phyla, we will refer to our groups as operational taxonomic units (OTUs). However, for simplicity we will still refer to our tree as the tree of bacterial phyla. At the time of the writing of this manuscript (April 2018), there were approximately 9,000 complete genomes available for bacteria at NCBI. In order to validate our results, two other datasets of 100 ( 381,000 protein sequences) and 400 complete genomes $(\sim 1.5 \mathrm{M}$ protein sequences) were downloaded and examined. All three datasets used were chosen randomly (except for the two phyla with only a single genome) and represented the phyla in proportion to the number of genomes available at NCBI.

Clustering, an important initial step in our analysis, is a popular approach in both computer science and statistics to find objects in a given set of data that can be grouped together to reduce complexity and to facilitate analysis. The goal is to divide the data into homologous subsets. For biological datasets, the clustering process typically begins with defining homology. For protein sequences, similarity (homology) can be measured using maximum alignment of the amino acids. In this study, we used a desktop version of the pClust software [14] for clustering. pClust uses a two-step process. In the first step pairs of sequences unlikely to have homologs are filtered out, and then pairwise semi-global alignment (default setting) is used to align the remaining pairs. The output of the first step is an undirected graph of nodes and edges that are used as the input into the second step [5]. In the second step the Louvain community detection algorithm is applied to this graph to detect clusters [15]. The algorithm finds clusters by optimizing a modularity metric. pClust yielded 61,057 non-singleton clusters (clusters with more than one protein sequence). Singletons, clusters with only one protein sequence, are excluded from the analysis carried out in this study.

\subsection{Constructing the Network of Organisms}

We used the protein clusters produced by pClust as a basis for understanding the relationships between organisms. Towards this end, we first modeled the organism-cluster relationship in a bipartite graph. In particular, we constructed a bipartite graph $G_{b}=\left(V_{1}, V_{2}, E\right)$, where $V_{1}$ corresponds to the organisms, $V_{2}$ corresponds to the clusters, and an edge $(u, v)$ exists if at least one protein sequence from organism $u$ is contained in cluster $v$. Figure 1a illustrates such a bipartite graph. For example, the edge $(\operatorname{Org} 1, C l 1)$ shows that a protein sequence from $\operatorname{Org} 1$ is contained in cluster $C l 1$. The three colors representing the organisms (red for Org1 and Org2; blue for Org3 and Org4; and yellow for Org5) indicate three distinct OTUs.

The network of organisms we sought (denoted as $G_{o}=\left(V_{1}, E^{\prime}, w\right)$ ) is derived from the bipartite graph $G_{b}$. In particular, each vertex in the weighted network $G_{0}$ is an organism, and two organisms are connected by an edge of weight $w$ that corresponds to the distance between the organisms in terms of their cluster memberships expressed in $G_{b}$. Specifically, the distance (dissimilarity) between the organisms is measured using the Manhattan distance [3] between them. The Manhattan distance evaluates the distance of one organism to another by measuring the number of changes from 0 to 1 or from 1 to 0 needed to transform the cluster membership indicator vector of the one organism to that of the other. If $p$ and $q$ are the cluster-membership vectors (of length $n$ ) corresponding to two organisms, the Manhattan distance between them is given by

$$
d(p, q)=\|p-q\|=\sum_{i=1}^{n}\left|p_{i}-q_{i}\right|
$$

Figure $1 \mathrm{~b}$ illustrates the network of organisms derived from the bipartite graph shown in Fig. 1a. In this example, the existence of 


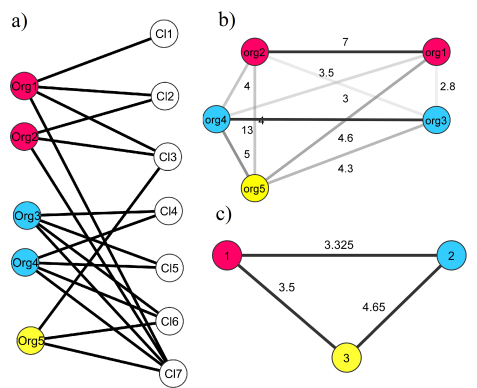

Figure 1: a) Example showing the bipartite graph of the organism-cluster relationship: Left side represents organisms and right side clusters. Nodes are colored according to phyla of the organisms. b) Network of organisms derived from graph in a). c) Network of phyla created from network in b).

clusters such as $\mathrm{Cl} 1$ and $\mathrm{Cl} 7$ implies that the Manhattan distance between every pair of organisms is non-zero, i.e., the network is a clique. The cluster $C l 1$ represents a singleton cluster that contains a protein sequence shared by only one organism, and the cluster $\mathrm{Cl7}$ represents the essential proteins shared by most of the organisms. In the illustration in Fig. 1b, the edge weights are similarities (which are obtained by reciprocating distances).

The weighted network of organisms $G_{o}$ can equivalently be viewed in terms of its adjacency matrix where the $(i, j)$ th entry shows the value of the Manhattan distance between organisms $i$ and $j$. We denote the adjacency matrix by OrganismMatrix. In order to obtain acceptable resolution in building the network from the distance matrix, we needed to normalize the entries of the matrix. Normalization was achieved by dividing each matrix entry by the row sum. Furthermore, to convert the distance (dissimilarity) into similarity, the reciprocal of each normalized entry was used. In the end, the normalized matrix corresponding to the network of organisms was achieved as follows:

$$
\text { OrganismMatrix }[i][j]_{\text {Norm }}=\frac{\sum_{q=1}^{n} \text { OrganismMatrix }[i][q]}{\text { OrganismMatrix }[i][j]}
$$

The matrix OrganismMatrix was visualized using the visone [1] software package. Because the resulting matrix was asymmetric, we did the computation for the upper triangle $(j>i)$ and then copied it to the lower triangle to make the matrix symmetric. The network corresponding to OrgamismMatrix was typically a clique. Therefore, for further analysis the network needed to be sparsified. We used the tools available in visone to sparsify the network.

\subsection{Sparsification}

Given an edge-weighted graph, sparsification produces a subgraph with fewer edges while maintaining structural and statistical properties of interest of the original graph [9]. In other words, sparsification finds the backbone of a network by removing "weak" links and reducing the complexity of the network while preserving its accuracy in capturing essential elements of the original dataset. For example, if a certain node has the highest weighted degree in the original network, it remains as the node with highest weighted degree in the sparsified network. Experimental results have shown that for many similarity networks in biological applications, important network properties can be maintained while sparsifying to about $20 \%$ of the original network $[9,13]$. Thus, for this work, we chose to keep the top $20 \%$ of the edges.

\subsection{Constructing the Network of OTUs}

The network of OTUs, which is also edge-weighted, was obtained from the network of organisms by calculating the average similarity between every pair of OTUs that were connected in the network of organisms. Specifically, let $s\left(x_{i}, x_{j}\right)$ be the similarity between organism $i$ and organism $j$ and let $M$ denote the set of edges connecting the two OTUs. The average similarity between the two OTUs is computed as follows:

$$
\text { AveSimilarity }=\frac{\sum_{\left(x_{i}, x_{j}\right) \in M} s\left(x_{i}, x_{j}\right)}{|M|}
$$

Figure 1c illustrates the network of OTUs created from the network of organisms in part b. For example, the similarity between the OTUs $P h 1$ and $P h 2$ is the average of the similarities represented by edges connecting organisms that belong to phylum $P h 1$ to organisms that belong to phylum/class $P h 2$. AveSimilarity $P h 1-P h 2=$ $1 / 4 \cdot(s(\operatorname{Org} 1, \operatorname{Org} 3)+s(\operatorname{Org} 1, \operatorname{Org} 4)+s(\operatorname{Org} 2, \operatorname{Org} 3)+s(\operatorname{Org} 2, \operatorname{Org} 4))=$ $1 / 4 \cdot(2.8+3.5+4+3)=3.325$

Similar to the adjacency matrix of the network of organisms, we worked with the adjacency matrix of the network of OTUs. The matrix was denoted as PhylaMatrix. The network of OTUs was visualized using the visone software package. Once again, the resultant network was found to be too dense to discern meaningful structure. In particular, the network was found to be essentially a complete graph. We therefore applied a weight threshold to remove low-weight edges and to sparsify the network. Specifically, we applied $20 \%$ sparsification using the backbone layout of visone.

\subsection{Creating the Tree of Bacterial Phyla}

To extract the tree of phyla from the network of OTUs, the shortest path from a particular source node in terms of graph distance must be calculated. A breadth-first search (BFS) is a suitable graph traversal procedure for achieving this [12]. Given a graph $G=(V, E)$ and a source node $s \in V$, a BFS is a full exploration of $G$ that produces a spanning tree of the graph containing all the edges that can be reached from $s$ and the shortest path from $s$ to each one of them. We applied a BFS to the network of OTUs using each of the OTUs in succession as the source node to determine the most plausible root of the tree.

\section{RESULTS}

\subsection{Network of Organisms}

Figure 2 shows the organism network for the 210 organisms in our study. Each node represents an organism, and the nodes are colored based on their OTUs. The original network was essentially a complete graph due to the existence of clusters of essential proteins that were present in all organisms. We therefore sparsified the network. Figure 2 shows the network after $20 \%$ sparsification.

The network shows that organisms from the same phylum/class tend to cluster together. Furthermore, in the network, it can be 


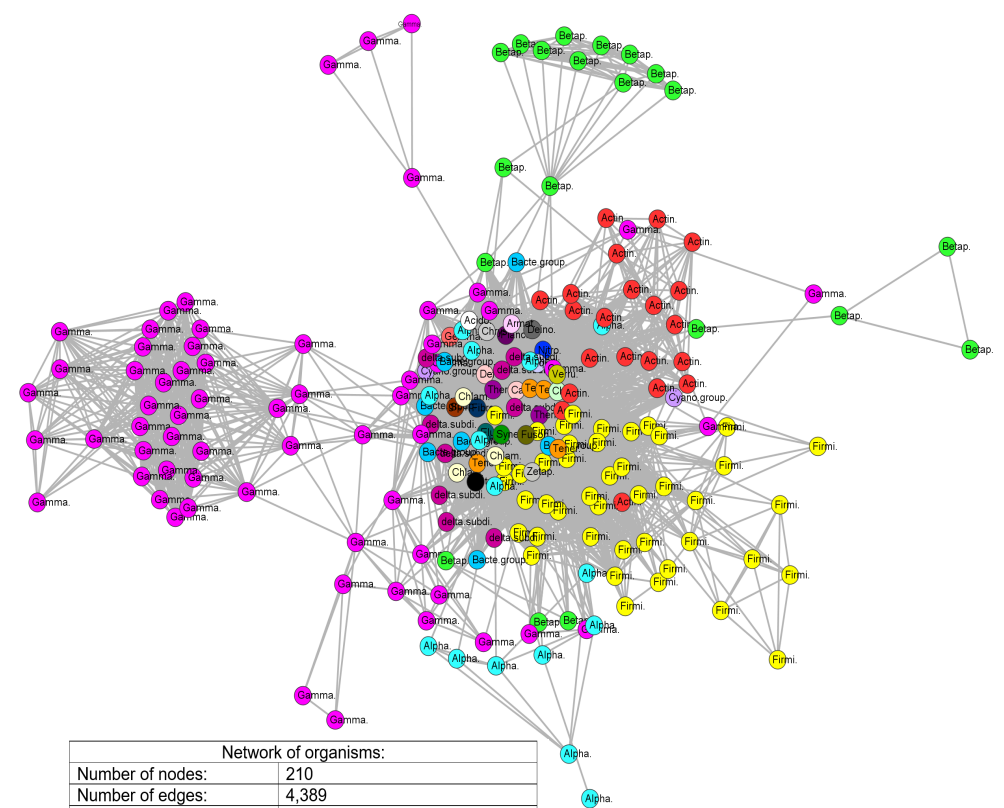

Figure 4: Network of phyla obtained after sparsification to $20 \%$. Each node color represents a phylum/class. Boxed values are key structural statistics.

Figure 2: Network of organisms obtained after sparsification to $20 \%$. Each node color represents a phylum/class. Node labels are the first five letters of the phylum/class name. Boxed values are key structural statistics.
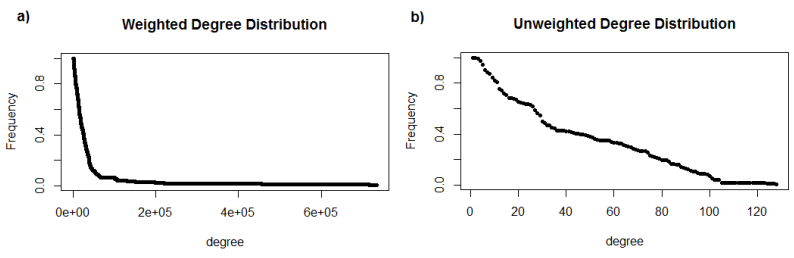

Figure 3: Degree distribution of the organism network: a) with edge weights and $b$ ) unweighted.

seen that weak ties (low edge weights) generally link two groups of OTUs, while strong ties (high weights) link organisms from the same phylum/class. This behavior is similar to the illustration in Fig. $1 \mathrm{~b}$ in which $\operatorname{Org} 1$ has a strong tie to $\operatorname{Org} 2$ while it has a weak tie to Org3. Figure 3 shows the degree distribution for the network in the case when weights are considered and the case when they are disregarded.

\subsection{Network of Bacterial Phyla}

Figure 4 shows the network of OTUs extracted from the network of organisms. Each node in the graph indicates a phylum/class. As we discussed before, there is an edge between two OTUs if their organisms share proteins. The network of OTUs was originally a hairball. The weak ties were removed from the network for further analysis. Figure 4 represents the network after removal of $80 \%$ of the weak links from the network.

\subsection{Tree of Bacterial Phyla}

The objective of this study is to determine the ancestral phylum of the tree of bacterial phyla which is equivalent to finding the source node that is the most plausible root of the tree. As discussed earlier, applying a BFS to the network of OTUs (Fig. 4) allows extraction of a tree of bacterial phyla based on the distance from a source node. Using a specific phylum/class as the source node results in a corresponding specific tree. Two examples are shown in Figs. 5 and 6. Figure 5 displays a tree of phyla with the Bacteroidetes/Chlorobi group as the source node, and Fig. 6 represents the tree starting from Gammaproteobacteria as the source node. The trees were found to have a star or star-like pattern. In fact, when Betaproteobacteria or Gammaproteobacteria was specified as the source, the tree was a star, and the distance of all other phyla from the source was a single hop. Another interesting finding is that when other nodes were considered as the source, the next level was usually Betaproteobacteria or Gammaproteobacteria, and the source was connected to all other phyla in two hops. In other words, the source reached the other nodes via Betaproteobacteria or Gammaproteobacteria.

Figure 5 shows that when the Bacteroidetes/Chlorobi group was the source, then Gammaproteobacteria, Betaproteobacteria, Actinobacteria, Alphaproteobacteria, Cyanobacteria, and Firmicutes could be reached in one hop, and the remaining OTUs were reachable from the source in two hops via these six OTUs. When Gammaproteobacteria is used as the source (root), Fig. 6 shows that all leaves are directly reachable (in one hop). 


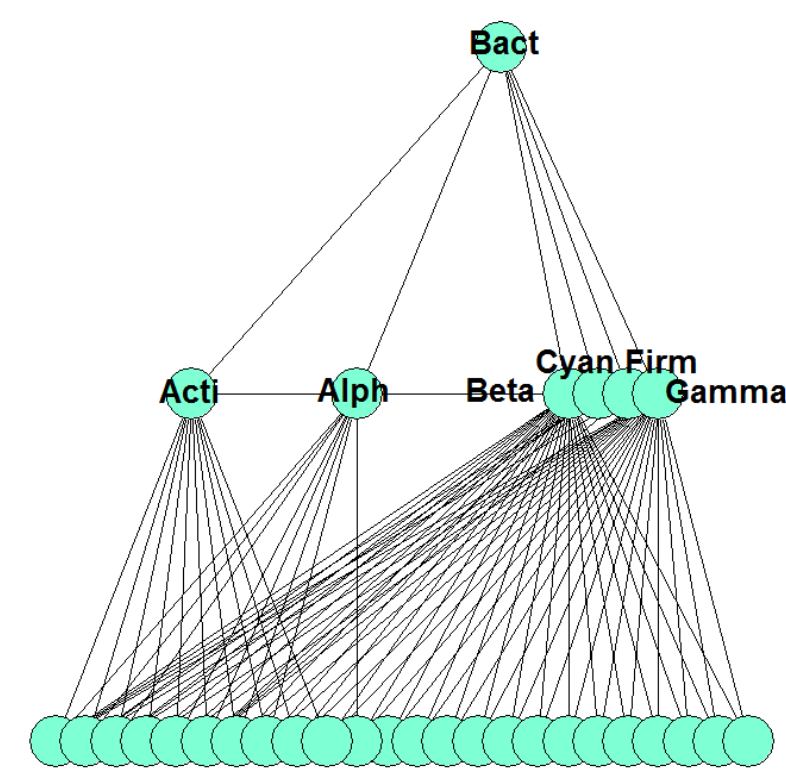

Figure 5: Tree of phyla with source node at the Bacteroidetes/Chlorobi group. The source node is at the first level, the next level shows phyla that are one hop from the source, and leaves represent phyla two hops from the source. Labels are OTU abbreviations.

To further show the star-like nature of all the possible trees, we created a matrix of shortest paths from the source node to all other nodes for the 31 source nodes. Table 1 shows the matrix for a select few of the 31; the entire matrix could not be listed due to space limitations. We also listed the sum and maximum of the results for each OTU, i.e., each row. The sum gives the summation of the shortest paths to all other nodes from the source node, and the maximum gives the height of the tree, i.e., the number of hops to a leaf. We see that Betaproteobacteria and Gammaproteobacteria have the smallest sum and the smallest maximum number of hops.

In addition to the $20 \%$ sparsification rate, we examined other rates. For a $10 \%$ sparsification rate only Gammaproteobacteria created a star, while using a $50 \%$ sparsification rate showed that Actinobacteria, Alphaproteobacteria, Betaproteobacteria, Cyanobacteria/Melaina bacteria group, Firmicutes, and Gammaproteobacteria generated stars. Interestingly, these latter OTUs were from the second level of the tree of phyla in Fig. 5.

\subsection{Centrality Analysis of the Network of OTUs}

Our final analysis identified the most important organisms and OTUs. We applied degree and betweenness centrality measures to both the network of OTUs and the network of organisms. Degree centrality identifies OTUs with the maximum number of connections (shared proteins). Betweenness centrality identifies OTUs that appear the most number of times acting as bridges along the shortest paths between pairs of OTUs. Both centrality measures indicated Buchnera aphidicola (Gammaproteobacteria: Enterobacteriaceae)

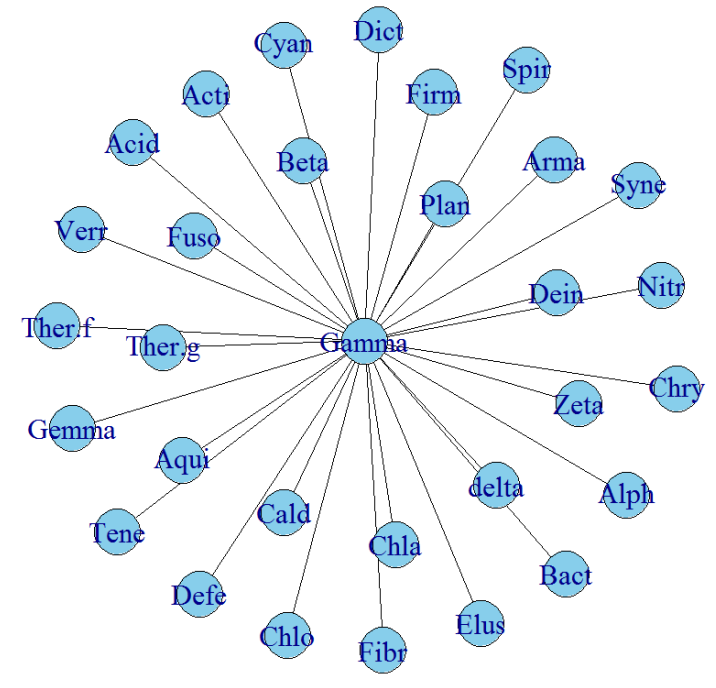

Figure 6: Tree of phyla with source node at Gammaproteobacteria. Nodes represent phyla, and labels are OTU abbreviations. The maximum path length from the source to any phylum was one hop.

Table 1: Matrix of shortest path lengths for recognized phyla. Partial results from the matrix are shown. The last two columns give the sum of all shortest path lengths for each phylum and the maximum shortest path length in a row, respectively. The maximum shortest path length is equivalent to the height of the tree.

\begin{tabular}{lccccc|cc}
\hline & Actin & Alpha & Aquif & Beta & Gamm & Sum & Max \\
\hline Actin & 0 & 1 & 2 & 1 & 1 & 44 & 2 \\
Alpha & 1 & 0 & 2 & 1 & 1 & 46 & 2 \\
Aquif & 2 & 2 & 0 & 1 & 1 & 58 & 2 \\
Beta & 1 & 1 & 1 & 0 & 1 & 30 & 1 \\
Gamm & 1 & 1 & 1 & 1 & 0 & 30 & 1 \\
\hline
\end{tabular}

as the most important organism in the network of organisms. $B$. aphidicola is the primary endosymbiont of almost all aphid species, and it inhabits specialized aphid cells called bacteriocytes [21]. Figure 7 visualizes the results for degree centrality for the network of OTUs. The results we obtained using betweenness centrality were similar to those obtained using degree centrality. Both Betaproteobacteria and Gammaproteobacteria had the maximum degree and, thus, were most important in the network of OTUs.

\section{DISCUSSION AND CONCLUSIONS}

The availability of an accurate and powerful clustering program together with the current availability of genomes from the majority of recognized phyla allowed us to use network science techniques to determine the possible ancestral phylum, i.e., the root of the tree of phyla. More than 700,000 protein sequences deduced from 210 completely sequenced bacterial genomes were sequenced. These genomes represented 28 of the 29 recognized bacterial phyla. 


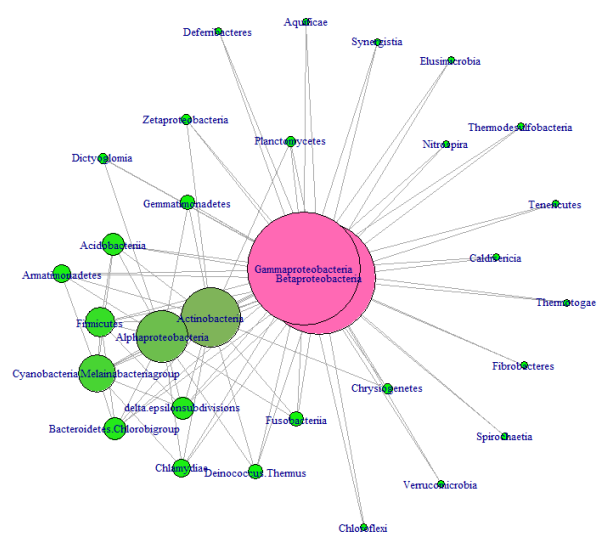

Figure 7: Visualization of the network of phyla with nodes sized according to their degree centrality: larger sizes indicate larger degrees.

A breadth-first search revealed two classes of the Proteobacterial phyla, Betaproteobacteria and Gammaproteobacteria, to be the oldest OTUs in the tree of phyla which makes Proteobacteria the ancestral phyla. Two centrality measures showed B. aphidicola, an endosymbiont belonging to Gammoproteobacteria, to be the most important organism in the network of organisms with more links to every other organism in the network than any other organism, further substantiating Gammaproteobacteria's place in the ancestral position. B. aphidicola has a small genome $(<1 \mathrm{Mb})$ with a low $\mathrm{G}+\mathrm{C}$ content, and it is thought that its ancestors were free living bacteria similar to modern Enterobacteraceae [7]. It is interesting that an organism with a reduced genome would be the most central because theoretically such an organism would have fewer opportunities to "make links" with other organisms in the network. This suggests that the core genome of B. aphidicola retains genes/proteins that are widely distributed across the phyla and that are relatively well conserved, while those genes/proteins that were lost during genome reduction are less important for understanding the broad relationships of the phyla. These "lost" genes/proteins include enzymes involved in the biosynthesis of non-essential amino acids, phospholipids, lipopolysaccharides, and genes/proteins involved in cellular defense [18]. B. aphidicola genomes have approximately 560 protein coding genes, and at the time of sequencing the first genome all but 83 of the predicted CDSs could be functionally assigned, indicating their conservation [18]. The Mycoplasma genitalium genome encodes even fewer proteins (470) than that of $B$. aphidicola and is thought to approach the minimal gene set [8]. The proteome of $M$. genitalium was not included in our study, but it would be interesting to see whether the organism also scores high in measures of centrality. If so, it would indicate that a small gene complement was driving selection because M. genitalium is a member of the phylum Tenericutes rather than Proteobacteria. In the future, we will refine our approach to determine which of the two classes, Betaproteobacteria or Gammaproteobacteria, is older.

\section{ACKNOWLEDGMENTS}

This work was supported by the National Science Foundation under the Advances in Biological Informatics program, Award 1262664.

\section{REFERENCES}

[1] Michael Baur, Marc Benkert, Ulrik Brandes, Sabine Cornelsen, Marco Gaertler, Boris Köpf, Jürgen Lerner, and Dorothea Wagner. 2001. Visone Software for visual social network analysis. In International Symposium on Graph Drawing. Springer, 463-464.

[2] Ludmila Chistoserdova. 2016. Wide distribution of genes for tetrahydromethanopterin/methanofuran-linked $\mathrm{C} 1$ transfer reactions argues for their presence in the common ancestor of bacteria and archaea. Frontiers in microbiology 7 (2016), 1425.

[3] Seung-Seok Choi, Sung-Hyuk Cha, and Charles C Tappert. 2010. A survey of binary similarity and distance measures. Fournal of Systemics, Cybernetics and Informatics 8, 1 (2010), 43-48.

[4] Francesca D Ciccarelli, Tobias Doerks, Christian Von Mering, Christopher J Creevey, Berend Snel, and Peer Bork. 2006. Toward automatic reconstruction of a highly resolved tree of life. science 311, 5765 (2006), 1283-1287.

[5] Jeff Daily. 2016. Parasail: SIMD C library for global, semi-global, and local pairwise sequence alignments. BMC bioinformatics 17, 1 (2016), 81.

[6] Frédéric Delsuc, Henner Brinkmann, and Hervé Philippe. 2005. Phylogenomics and the reconstruction of the tree of life. Nature Reviews Genetics 6, 5 (2005), 361.

[7] AE Douglas. 1998. Nutritional interactions in insect-microbial symbioses: aphids and their symbiotic bacteria Buchnera. Annual review of entomology 43, 1 (1998), $17-37$.

[8] Claire M Fraser, Jeannine D Gocayne, Owen White, Mark D Adams, Rebecca A Clayton, Robert D Fleischmann, Carol J Bult, Anthony R Kerlavage, Granger Sutton, Jenny M Kelley, et al. 1995. The minimal gene complement of Mycoplasma genitalium. Science 270, 5235 (1995), 397-404.

[9] Michael Hamann, Gerd Lindner, Henning Meyerhenke, Christian L Staudt, and Dorothea Wagner. 2016. Structure-preserving sparsification methods for social networks. Social Network Analysis and Mining 6, 1 (2016), 22.

[10] Laura A Hug, Brett J Baker, Karthik Anantharaman, Christopher T Brown, Alexander J Probst, Cindy J Castelle, Cristina N Butterfield, Alex W Hernsdorf, Yuki Amano, Kotaro Ise, et al. 2016. A new view of the tree of life. Nature microbiology 1 (2016), 16048

[11] Erich D Jarvis, Siavash Mirarab, Andre J Aberer, Bo Li, Peter Houde, Cai Li, Simon YW Ho, Brant C Faircloth, Benoit Nabholz, Jason T Howard, et al. 2014. Whole-genome analyses resolve early branches in the tree of life of modern birds. Science 346, 6215 (2014), 1320-1331.

[12] Charles E Leiserson and Tao B Schardl. 2010. A work-efficient parallel breadthfirst search algorithm (or how to cope with the nondeterminism of reducers). In Proceedings of the twenty-second annual ACM symposium on Parallelism in algorithms and architectures. ACM, 303-314.

[13] Gerd Lindner, Christian L Staudt, Michael Hamann, Henning Meyerhenke, and Dorothea Wagner. 2015. Structure-preserving sparsification of social networks. In Proceedings of the 2015 IEEE/ACM International Conference on Advances in Social Networks Analysis and Mining 2015. ACM, 448-454.

[14] Svetlana Lockwood, Kelly A Brayton, and Shira L Broschat. 2016. Comparative genomics reveals multiple pathways to mutualism for tick-borne pathogens. BMC genomics 17, 1 (2016), 481.

[15] Hao Lu, Mahantesh Halappanavar, and Ananth Kalyanaraman. 2015. Parallel heuristics for scalable community detection. Parallel Comput. 47 (2015), 19-37.

[16] Aidan C Parte. 2013. LPSNâĂŤlist of prokaryotic names with standing in nomenclature. Nucleic acids research 42, D1 (2013), D613-D616.

[17] Frederik Schulz, Emiley A Eloe-Fadrosh, Robert M Bowers, Jessica Jarett, Torben Nielsen, Natalia N Ivanova, Nikos C Kyrpides, and Tanja Woyke. 2017. Towards a balanced view of the bacterial tree of life. Microbiome 5, 1 (2017), 140.

[18] Shuji Shigenobu, Hidemi Watanabe, Masahira Hattori, Yoshiyuki Sakaki, and Hajime Ishikawa. 2000. Genome sequence of the endocellular bacterial symbiont of aphids Buchnera sp. APS. Nature 407, 6800 (2000), 81.

[19] Madeline C Weiss, Filipa L Sousa, Natalia Mrnjavac, Sinje Neukirchen, Mayo Roettger, Shijulal Nelson-Sathi, and William F Martin. 2016. The physiology and habitat of the last universal common ancestor. Nature Microbiology 1, 9 (2016), 16116.

[20] Carl R Woese and George E Fox. 1977. Phylogenetic structure of the prokaryotic domain: the primary kingdoms. Proceedings of the National Academy of Sciences 74, 11 (1977), 5088-5090.

[21] Ting-Ting Xu, Jing Chen, Li-Yun Jiang, and Ge-Xia Qiao. 2017. Historical and cospeciating associations between Cerataphidini aphids (Hemiptera: Aphididae: Hormaphidinae) and their primary endosymbiont Buchnera aphidicola. Zoological Journal of the Linnean Society (2017). 